\title{
Adaptive Radiotherapy for Glioblastoma Multiforme - The Impact on Disease Outcome
}

\author{
ZOLTÁN VÉGVÁRY ${ }^{1}$, BARBARA DARÁZS ${ }^{1}$, VIKTOR PACZONA ${ }^{1}$, ÁGNES DOBI ${ }^{1}$, \\ ZITA REISZ ${ }^{3}$, ZOLTÁN VARGA ${ }^{1}$, EMESE FODOR ${ }^{1}$, ADRIENN CSERHÁTI ${ }^{1}$, \\ JUDIT OLÁH ${ }^{1}$, DÁVID KIS ${ }^{4}$, PÁL BARZÓ ${ }^{4}$ and KATALIN HIDEGHÉTY ${ }^{1,2}$ \\ ${ }^{1}$ Department of Oncotherapy, University of Szeged, Szeged, Hungary; \\ ${ }^{2}$ ELI-ALPS Non-profit Ltd, Szeged, Hungary; \\ ${ }^{3}$ Department of Pathology, University of Szeged, Szeged, Hungary; \\ ${ }^{4}$ Department of Neurosurgery, University of Szeged, Szeged, Hungary
}

\begin{abstract}
Background/Aim: To study the changes of glioblastoma multiforme during chemoradiotherapy (CRT) and to evaluate the impact of changes on dosimetry and clinical outcomes. Patients and Methods: Forty-three patients underwent volumetric imaging-based replanning. Prognostic factors and gross tumor volume changes in relation to overall survival and the effect of adaptive replanning were statistically analyzed. Results: Patients with total tumor removal, with shorter time to $C R T$ ( $<27$ days), with methylated O-6 methylguanine DNA methyltransferase and good performance status (>60\%) had better survival. Tumor shrinkage in 24 patients resulted in improved survival compared to 19 in whom tumor was unchanged or progressed (25.3 vs. 11.1 months, $p=0.04)$. Adapted planning target volume allowed a reduction in irradiated volume, while increasing survival (12.06 vs. 28.98 months, $p=0.026)$. Conclusion: Tumor response during CRT has significant impact on the outcome. Adaptation of the planning target volume to the tumor changes proved to be beneficial and warrants further investigation.
\end{abstract}

The advent of various forms of high-tech volumetric imaging has opened up the possibility of defining and following target and normal structures in the brain with high resolution prior to and during a course of radiation therapy (RT). Enhanced dose-delivery methods using new generation linear accelerators (LINAC) or increasingly available nuclear particle accelerators allow highly selective dose distribution for predefined structures (1-4). These developments can lead to a remarkably improved therapeutic ratio. Toward that

Correspondence to: Barbara Darázs, Department of Oncotherapy, University of Szeged, Szeged, Hungary. E-mail: darazsb@gmail.com

Key Words: Adaptive radiotherapy, replanning, gliomas, brain tumor. goal, many newly defined structures have to be delineated. Furthermore, if the volume and location of the primarily contoured structures change, particularly small structures (such as chiasma, subventricular zone (SVZ) and hippocampus), those should also be followed and the RT plan subsequently modified (5). Several studies have investigated spatial and dosimetric changes in critical structures during treatment for different cancer types (6-8), but much less research has been performed on RT of the brain, which has great anatomical constancy due to the closed skull volume and lack of organ movement. However, tumor volume, the surgical cavity, the peritumoral region and several sensitive brain structures are assumed to undergo slow but evident changes (e.g. hemorrhage, edema and shift of anatomical structures) owing to the development of radiation-related reactions and residual tumor response (912). The importance of such anatomical changes during the course of RT increases if growing numbers of small substructures [target and organs at risk (OARs)] are defined for dose prescription. The standard OARs for brain tumor RT include the optic nerves, optic chiasm, eyes, lenses, brain and brainstem. Optionally, the cochleae, lacrimal glands, pituitary gland, hypothalamus and hippocampus could be taken into account for treatment planning when the tumor is in a location that will allow sparing without compromising the dose to the target (13-15).

Glioblastoma is a tumor that invades surrounding tissues aggressively, becomes infiltrative and spreads into different regions of the brain. Defining the clinical target volume (CTV) on postoperative images is therefore a highly challenging task (16-18). Preoperative contrast-enhancing volume cannot be directly used due to postoperative changes, and the resection cavity does not correspond accurately to the high number tumor cell region. Additionally, residual contrast-enhancing and non-enhancing tumor should be included in the CTV. Recently, advanced 
imaging techniques have been recommended to define the volume of the tumor mass, such as different sequences of magnetic resonance imaging (MRI) and amino acid-based positron-emission tomography images $\left({ }^{11} \mathrm{C}\right.$-methionine and O-(2- $\left[{ }^{18} \mathrm{~F}\right]$ fluoroethyl)-L-tyrosine) (17-19). Furthermore, different approaches to the definition of 'target volume' are practiced. The Radiation Therapy Oncology Group (RTOG) guidelines suggest two-phase irradiation, using larger CTV, including postoperative peritumoral edema, delineated on MRI T2-weighted fluid-attenuated inversion recovery (FLAIR) images, plus a $2 \mathrm{~cm}$ margin up to $46 \mathrm{~Gy}$, followed by boost therapy encompassing the residual tumor with an additional margin of $2 \mathrm{~cm}$ (20). In Europe, the European Organisation for Research and Treatment of Cancer (EORTC) consensus guideline recommends single-phase irradiation with a $20 \mathrm{~mm}$ margin around the gross tumor volume (GTV), defined as the resection cavity plus residual contrast-enhancing tumor enlarged with a 3-5 mm margin for institutional set-up uncertainties (21). A recent retrospective analysis of the recurrence pattern exhibited no significant difference using such a limited (EORTC) approach or even further reduced margins $(10 \mathrm{~mm}$ and 5 $\mathrm{mm}$, respectively) around the GTV for glioblastoma to create the CTV (22). Whether to incorporate pre/postoperative edema is also a subject of debate. In recent years, growing attention has been paid to the dose in the SVZ, the region around the lateral ventricles, postulated as a main niche of pluripotent neural stem cells of the central nervous system. These cells, with their capacity to act as tumor stem cells, are able to differentiate into neurons or glial cells and serve as a source of tumor development and recurrence. Based on retrospective dose distribution analyses, a high dose to the ipsilateral SVZ resulted in significant improvement of progression-free (PFS) and overall (OS) survival for patients with glioblastoma (23-29). Therefore, the inclusion of the ipsilateral SVZ into the CTV may be considered.

The target volume definition of GBM varies remarkably at different institutions worldwide as a result of contradictory recommendations. The use of several MRI sequences at different time points, including preoperative MRI with a diffusion-weighted sequence for tractography and functional MRI, thereafter MRI within 48 hours post-surgery and a further pre-RT MRI, has recently become a standard requirement (30).

We investigated the potential of the use of repeated CT/MRI during two-phase RT delivery and adaptation of the structure definition for replanning and its impact on survival.

\section{Patients and Methods}

Study population. Forty-three consecutive patients with GBM treated at the Department of Oncotherapy University of Szeged,
Table I. Patient characteristics.

\begin{tabular}{lc}
\hline Characteristic & Frequency, $\mathrm{n}$ \\
\hline Gender & \\
Male & 19 \\
Female & 24 \\
Age & \\
$\geq 60$ Years & 22 \\
$\quad<60$ Years & 21 \\
Histology & \\
Glioblastoma & 43 \\
Type of surgery & \\
Biopsy & 7 \\
Subtotal resection & 27 \\
Gross total resection & 9 \\
KPS & \\
$>60 \%$ & 16 \\
$\leq 60 \%$ & 27 \\
MGMT status & \\
$>40 \%$ & 17 \\
$\leq 40 \%$ & 16 \\
Unknown & 10 \\
RT start & \\
$<27$ Days & 22 \\
$\geq 27$ Days & 21 \\
Therapy & \\
Chemoradiotherapy & 39 \\
Radiotherapy alone & 4 \\
\hline
\end{tabular}

KPS: Karnofsky Performance Status; MGMT: O-6 methylguanine DNA methyltransferase promoter methylation; RT start: time interval between the surgery and the radiotherapy start date.

Hungary, between January 2013 and June 2016 were selected for a retrospective study. The patient and tumor characteristics as well as the volumetric data for the defined targets are provided in Tables I and II. The average age of the 43 patients ( 19 males and 24 females) was 58.6 (range=12-85) gears. Thirty-nine patients were treated with concurrent temozolomide chemotherapy during RT followed by temozolomide monotherapy, and four patients received only RT. All the patients underwent surgical tumor removal, with the tumor type confirmed by histology. The extent of the tumor removal of the entire study group was by subtotal resection in the majority of cases $(\mathrm{N}=27)$. The $O-6$ methylguanine DNA methyltransferase (MGMT) status was available for 33/43 tumor samples for the present analysis and 17 were defined as being promoter region methylated by immunohistochemistry. The average time to planning $\mathrm{CT}$ after surgery was 2.8 (range=0.7-5.1) weeks. RT generally started 1 week after the planning $\mathrm{CT}$, thus the mean time interval between surgery and CRT was 29.1 days (range=5-59 weeks). GBM was treated with $75 \mathrm{mg} / \mathrm{m}^{2}$ temozolomide each day during RT, with a $60 \mathrm{~Gy}$ total dose administered in two phases $(40 \mathrm{~Gy}+$ repeated planning CT/MRI-based replanning of a 20 Gy boost) conventionally fractioned at $2 \mathrm{~Gy}$ per fraction. All the patients had an additional (secondary) replanning CT/MRI (mean=3.9, range=3.7-4.0 weeks) after the start of RT [mean=7.7 (range=5.3-14.3) weeks after surgery], which was registered to the initial (primary) planning CT. The University Ethics Committee and the local Institutional Review Board approved the study under registration no. 46/2015. 

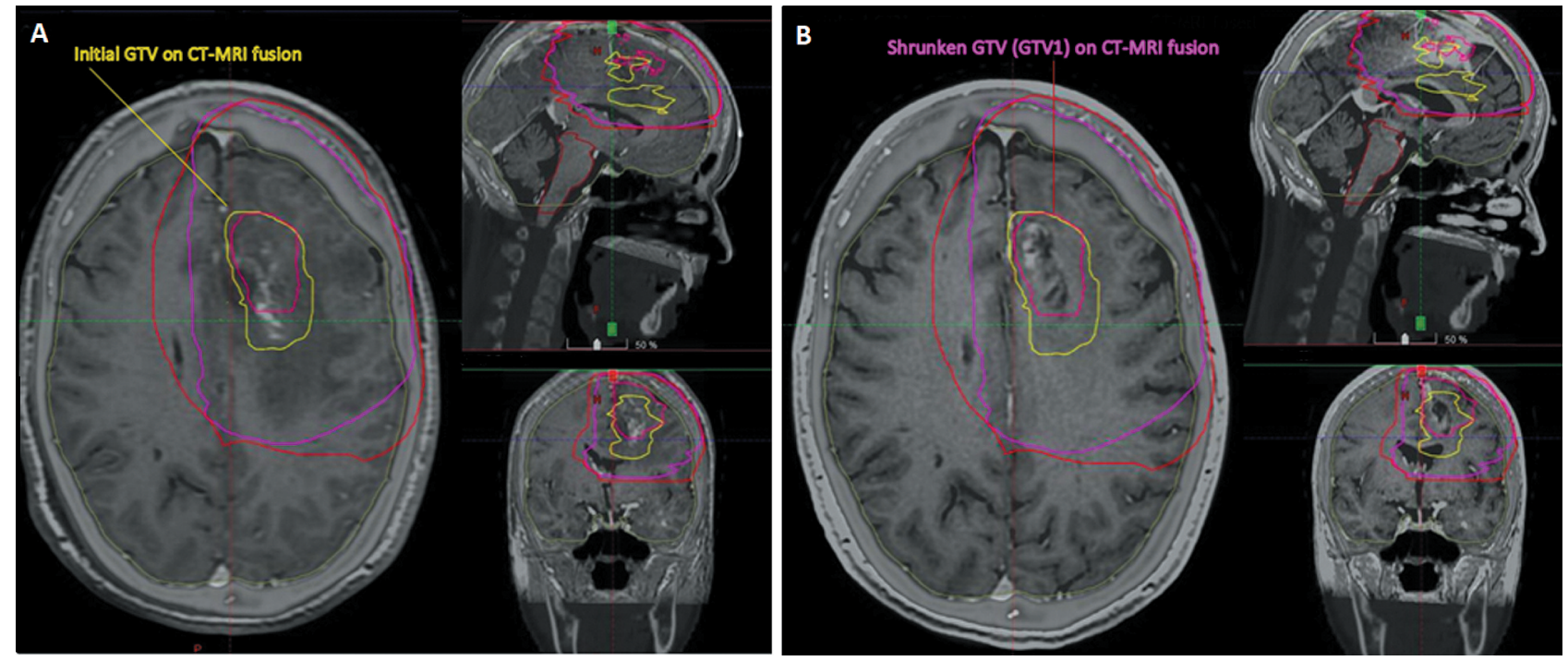

Figure 1. A: Initial gross tumor volume (GTV - yellow) and planning target volume (PTV - red) after 40 Gy. B: Irradiation target volumes were recontoured (GTV1 - pink; PTV1 - purple).

Table II. Gross tumor volume (GTV) and planning target volume (PTV) on primary and on secondary (GTV1 and PTV1) computed tomography and their difference. Data are the mean \pm standard deviation.

\begin{tabular}{lcccccc}
\hline Volume & GTV $\left(\mathrm{cm}^{3}\right)$ & GTV1 $\left(\mathrm{cm}^{3}\right)$ & $\Delta \mathrm{GTV}\left(\mathrm{cm}^{3}\right)$ & PTV $\left(\mathrm{cm}^{3}\right)$ & PTV1 $\left(\mathrm{cm}^{3}\right)$ & $\Delta$ PTV $\left(\mathrm{cm}^{3}\right)$ \\
\hline Overall & $98.9 \pm 67.4(\mathrm{n}=43)$ & $106.3 \pm 67.7$ & $6.7 \pm 2.7$ & $530.2 \pm 160.5(\mathrm{n}=43)$ & $359.9 \pm 125.2$ & $-183.2 \pm 130.5$ \\
Regression & $113.1 \pm 69.4(\mathrm{n}=24)$ & $85.5 \pm 56.9$ & $-27.56 \pm 20.8$ & $547.2 \pm 162.3(\mathrm{n}=41)$ & $353.6 \pm 122.8$ & $-193.6 \pm 124.4$ \\
No change/progression & $94.6 \pm 66.2(\mathrm{n}=19)$ & $113.5 \pm 75.5$ & $19.2 \pm 1.8$ & $460.0 \pm 114.5(\mathrm{n}=2)$ & $489.6 \pm 140.6$ & $29.6 \pm 26.0$ \\
\hline
\end{tabular}

$\Delta \mathrm{GTV}=\mathrm{GTV} 1-\mathrm{GTV} ; \Delta \mathrm{PTV}=\mathrm{PTV} 1-\mathrm{PTV}$.

Contouring and treatment planning. Patients were positioned and fixed using a 3-point individual thermoplastic mask, with a CT scan taken with slice thickness of $\leq 5 \mathrm{~mm}$ with the patient in a supine position. The GTV and planning target volume (PTV) were defined on the primary CT using pre- and postoperative MRI images. Registration and contouring were performed with Advantage SIM software (version 4.7; General Electric Healthcare, Chicago, IL, USA). Contouring was performed in axial reconstructions of the CT data set after MRI-CT image fusion. The PTV margin around the GTV on the preoperative gadolinium-enhanced T1-weighted MRI sequence was defined according to our Institutional protocol based on the RTOG contouring guidelines. Around the GTV, a $20 \mathrm{~mm}$ margin was created encompassing the peritumoral edema defined on the basis of postoperative T2-FLAIR MRI. In the case of excessive edema, the margin was adapted manually in individual cases. All plans were made and optimized in the Xio Planning System (version 4.7; Elekta, Stockholm, Sweden). The PTV was treated with 3-dimensional conformal RT or intensity-modulated RT (IMRT) up to $40 \mathrm{~Gy}$ in 20 fractions with regular position control using portal imaging or cone-beam CT. After the first period of study, a second planning CT or, more recently, MRI, was performed.
GTV1 and PTV1 were defined on the secondary planning CT. When an MRI was taken, image registration was applied for delineation of GTV1 and PTV1 on the planning CT (Figure 1). PTV1 was assigned by adding a $10 \mathrm{~mm}$ margin around GTV1. The PTV1 volume was treated with 3D-conformal RT/IMRT delivering an additional $20 \mathrm{~Gy}$ in 10 fractions.

Statistical analysis. The primary endpoint was OS and targetvolume parameters. OS was measured from the date of histological diagnosis to the date of death from any cause. Patients who developed none of these time-to-event endpoints were censored on the date of their last follow-up. Survival distributions and median survival data were estimated using the Kaplan-Meier method, and comparisons were performed based on the log-rank test for categorical characteristics. Cox proportional hazards regression models were fitted to examine the association of RT parameters with OS. Variables with $p$-values of less than 0.05 in the univariate analysis were selected for the multivariate Cox proportional hazards model. Statistical analysis was performed using the SPSS statistical analysis software package (version 20; IBM, Armonk, NY, USA). Statistical significance was set at a threshold of $p<0.05$. 

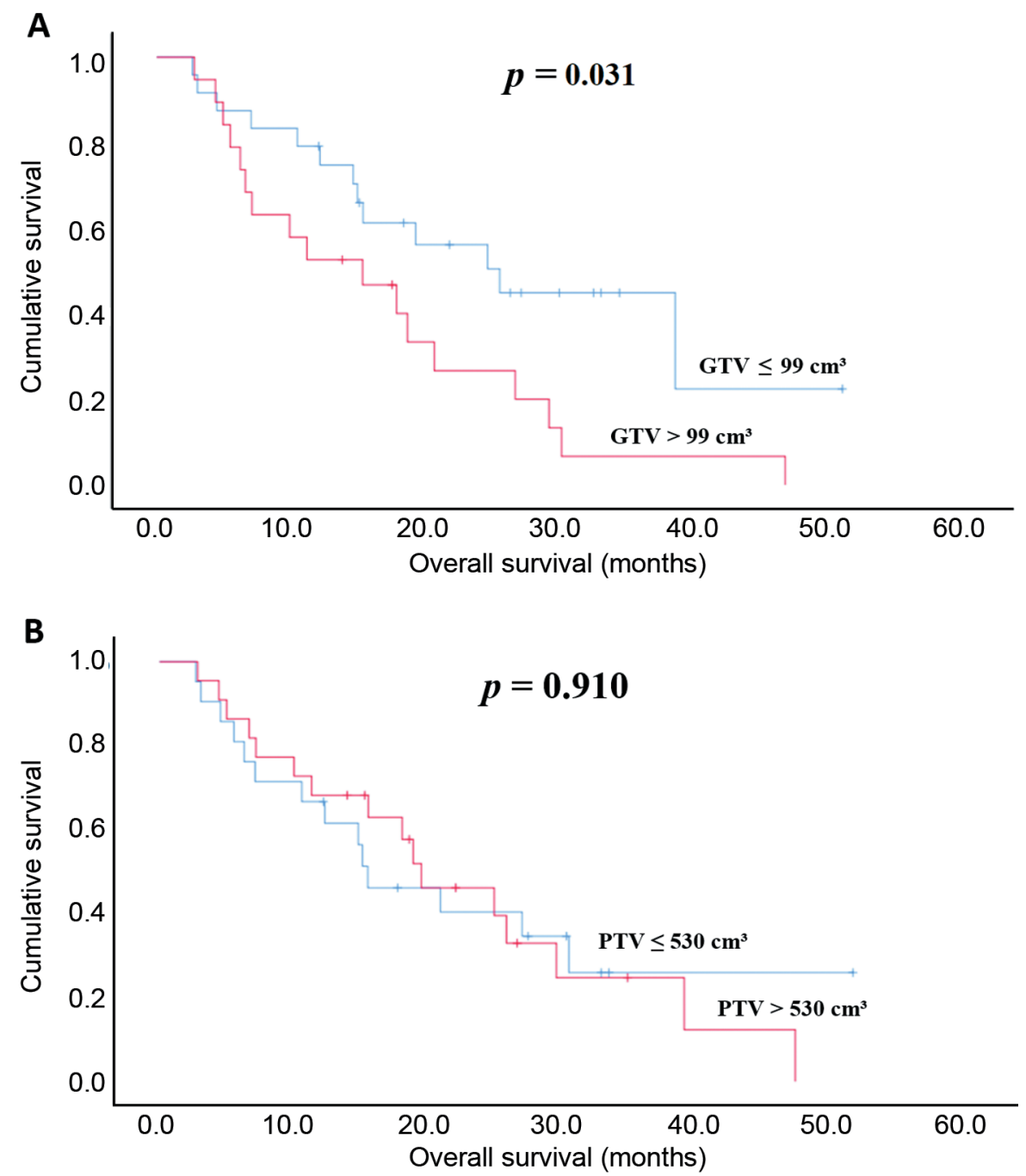

Figure 2. Correlation of initial gross tumor volume (GTV) (A) and initial planning target volume on primary (PTV) (B) on primary computed tomography with overall survival.

\section{Results}

The initial size of the GTV was strongly inversely correlated with OS. The patients were separated for the Kaplan-Meier analysise into two groups according to the mean GTV size: $\leq 99 \mathrm{~cm}^{3}$ and $>99 \mathrm{~cm}^{3}$. The median OS was 25.33 months (95\% CI=19.59-35.27 months) for the first group and 15.21 months (95\% CI=10.82-22.27 months) for the second, corresponding to a hazard ratio (HR) of $1.006(95 \%$ $\mathrm{CI}=1.00-1.01, p=0.031$ ) (Figure 2A). Using the average value as cut-off point, the PTV did not exhibit a correlation with survival. Median OS was 15.21 months (95\% $\mathrm{CI}=15.06-31.34$ months) in the first group and 19.12 months (95\% CI=15.64-28.67 months) in the second group corresponding to an $\mathrm{HR}$ of 1.001 (95\% CI=0.99-1.01, $p=0.910$ ) (Figure 2B).
Anatomical changes in the brain and tumor growth or shrinkage occurred during RT. The GTV volume change ( $\triangle \mathrm{GTV}=\mathrm{GTV} 1-\mathrm{GTV})$ during RT was correlated with OS. The median OS was 25.33 months $(95 \% \mathrm{CI}=21.68-35.28$ months) in the group with $\Delta \mathrm{GTV}<0 \mathrm{~cm}^{3}$, i.e. GTV regression, and 11.10 months $(95 \% \mathrm{CI}=10.63-22.69$ months) in the group with $\Delta \mathrm{GTV} \geq 0 \mathrm{~cm}^{3}$, i.e. no change or progression of GTV, corresponding to an HR of $1.006(95 \%$ $\mathrm{CI}=0.99-1.01, p=0.040$ ) (Figure 3A). The recontouring and change of PTV was significantly different between the two groups: Patients with $\triangle \mathrm{PTV} \leq 183 \mathrm{~cm}^{3}$ and those with $\triangle$ PTV $>183 \mathrm{~cm}^{3}$. The median OS was 12.06 months $(95 \%$ $\mathrm{CI}=11.63-22.91$ months) for the first group and 28.98 months (95\% CI=22.36-38.82 months) for the second, corresponding to an $\mathrm{HR}$ of 1.001 (95\% CI=0.99-1.01, $p=0.026$ ) (Figure 3B). 

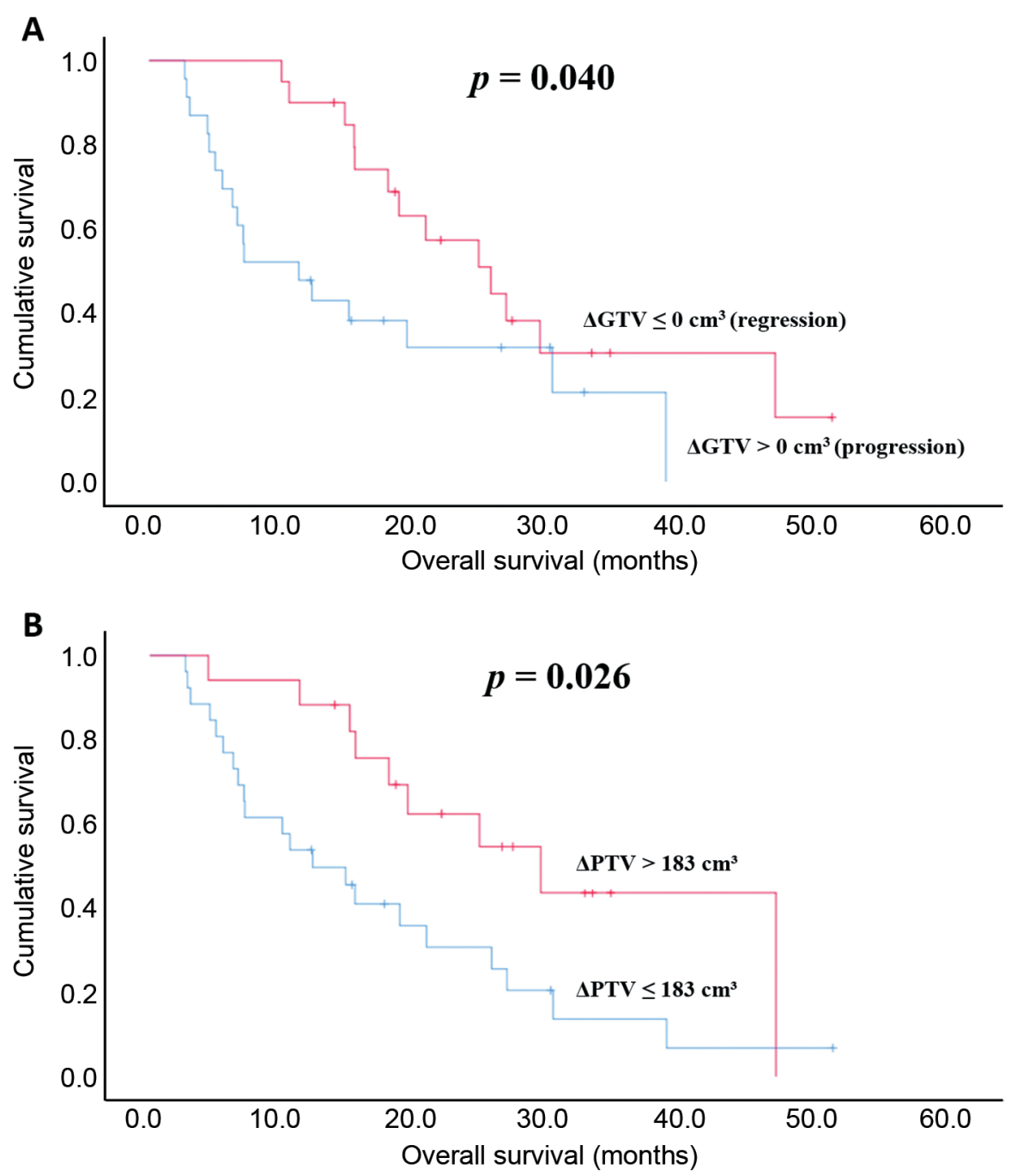

Figure 3. Correlation of change in gross tumor volume $(\triangle G T V)(A)$ and in planning target volume $(\triangle P T V)(B)$ with overall survival.

The type of surgery had a significant impact on survival, patients who underwent biopsy had a mean survival of 6.97 months, those with subtotal tumor resection 20.5 months and with gross tumor resection of 25.33 months $(p=0.009)$ respectively. A Kaplan-Meier analysis of the time from surgery to the start of CRT and OS revealed that CRT started within 27 days resulted in a significantly higher mean OS (26.48 vs. 15.21 months, $p=0.046$ ). Our results demonstrated that $M G M T$ promoter methylation was associated with significantly longer OS. The median OS was $7.03(95 \%$ $\mathrm{CI}=7.58$-18.44) months in those with non-methylated $M G M T$ promoter $(\leq 40 \%)$ and $26.48(95 \% \mathrm{CI}=19.54-35.86)$ months in those with $>40 \%$ MGMT promoter methylation, corresponding to an HR of 1.017 (95\% CI=0.99-1.03, $p=0.065)$, retrospectively. Patients with higher postoperative
Karnofsky Performance Status (KPS) status (KPS>60\%) also demonstrated increased OS, with 38.31 (95\% CI=27.4642.01) months versus 11.10 (95\% CI=10.11-17.75) months for the other group, corresponding to an HR of $0.949(95 \%$ $\mathrm{CI}=0.92-0.97, p<0.001)$.

\section{Discussion}

In accordance with the literature, our data confirm the impact of general prognostic factors on disease outcome, i.e. better KPS, larger extent of tumor removal and methylated MGMT promoter status resulted in longer survival. The optimal time interval between the surgery and start of CRT is a matter of debate in the literature (29) and a clear conclusion cannot be drawn in our patient group, although the shorter time to CRT 
proved to be significant factor for better OS. Adaptive RT has mainly been applied in patients with extra-cranial localization taking into account anatomical changes associated with weight loss, internal organ movement, organ filling, tissue edema and potential tumor regression. These changes may significantly influence the dose distribution, resulting in target volume missing or overdosing in the OAR region. The studies published on adaptive RT have varied between daily onboard imaging-based plan adaptation and CT/MRI-based replanning prior to boost definition. The final definition of the optimal time point and methodology for these resource-consuming procedures is yet to be determined. The advantageous effect of replanning for brain irradiation is not yet supported by strong clinical evidence despite the increased attention to adaptive techniques and a growing amount of clinical data for tumors in other locations. There are limited data available on adaptive RT including brain structures in publications on head and neck region RT. A study by Ho's team found no relevant effect of a daily assessment of dose-distribution changes for the brainstem and spinal cord in oropharyngeal cancer and hence did not recommend frequent replanning from this aspect (31). In contrast, numerous other studies have confirmed the benefit of adaptive RT for the treatment of head and neck tumors to outcomes (32-36). A recent study on adaptive RT of advanced head and neck cancer (36) demonstrated an improved therapeutic index by increasing the tumor coverage and dose reduction to the OARs. It confirmed that without replanning, the dose to some OARs would have exceeded their respective tolerance threshold, including central nerve system structures, i.e. the brainstem and spinal cord.

Unlike extra-cranial localizations, data on adaptive RT for brain tumors is sparse in spite of the fact that GBM is known as a rapidly growing tumor type, and CRT is applied postoperatively when relevant changes in post-surgical and tumor volume are supposed to occur $(5,9-12,37)$. Both internationally applied guidelines (RTOG and EORTC) define target volume based on surgical cavity, edema and residual tumor. However, neither of them contains a recommendation for CT/MRI-based replanning during the course of RT. According to the institutional strategy at our Oncotherapy Department, two-phase irradiation is planned with the shrinking-volume technique. A pre-therapeutic boost definition is applied in conjunction with a recontouring of the residual tumor mass (GTV2) for repeated planning CT/MRI in an adapted CTV2-PTV2 approach. Recently, an evaluation on inter-fractional variation for completely resected GBM has been reported. Surgical cavities of 19 patients with glioblastoma with gross total resection were measured at three time points, 1 day following surgery, 4 weeks thereafter at the planning of RT and 5 weeks later (after 50 Gy was delivered) prior to boost planning. The differences between the surgical defect volumes were statistically significant $(p<0.001)$, and based on the planning comparison, the authors concluded that the volume-adapted replanning during RT might reduce the irradiated volume of normal brain tissue and prevent a radiation target miss for boost RT (10). In line with this research, we detected relevant morphological changes on CT/MRI-based replanning performed prior to the boost irradiation. Moreover, patients were included with macroscopic tumors after partial resection and biopsy in which tumor response had contributed greatly to target volume changes in addition to post-surgical and RT-triggered reactions. In a preliminary study on three patients with GBM using integrated high-field MRI-LINAC, relevant volumetric changes in GBM tumor volume had been observed over the course of RT (37). Muruganandham et al. compared the status of tumor metabolic activity with MRI spectroscopy prior to and during the third week of RT, revealing a significant correlation with PFS (38). In our study, both the initial residual tumor volume and the extent of tumor shrinkage exhibited a significant impact on OS. The outcome of survival analysis showed no significant difference in terms of the initial size of the PTV. However, the GTV volume difference i.e. the difference between the GTV defined on the first plan and the tumorous mass seen on the replanning image, did show a significant correlation with OS in univariate analysis. Similarly, a relevant decrease in the size of the PTV (the PTV volume difference, analogous to the GTV volume difference) predicted better OS.

Our research has certain limitations due to its retrospective nature and relatively small number of patients. Thus, the correlation between the adapted boost volume and OS disappeared in the multivariate analysis, taking into account stronger prognostic factors, such as KPS and MGMT promoter methylation status. Furthermore, the first series of replanning took place using repeated CT images but in the later part of the study, all patients underwent repeated MRI. In order to compensate for the lack of MRI and increase the accuracy of the boost volume delineation, in all cases, two experts (one of them a neuro-radiologist) defined GTV1.

Our findings support the great importance of monitoring anatomical changes in the course of fractionated postoperative brain tumor irradiation. A follow-up of the residual tumor during CRT and adaptation of the PTV to tumor volume changes result in increased accuracy of dose delivery to the tumor and relevant normal brain tissue sparing. According to our data, reduction of the PTV did not compromise survival; in contrast, it seemed to be beneficial. Our preliminary data on improved survival on the basis of a higher degree of PTV reduction warrant further clinical studies to confirm these encouraging results.

The implementation of an adaptive RT approach is suggested for postoperative irradiation of GBM to optimize coverage of the target and minimize the dose to OARs. The reported data confirm that significant changes occur in different brain structures and in the residual tumor during 
fractionated CRT. The tumor response and adapted boost volume definition exhibited a strong correlation to treatment outcome. Optimization of the imaging (MRI and amino acidbased PET/CT) for replanning could further improve the quality of the adaptive approach.

\section{Conflicts of Interest}

None of the Authors has a financial or personal relationship with other people or organizations that could inappropriately influence or bias the content of the article.

\section{Authors' Contributions}

$\mathrm{KH}, \mathrm{A} \mathrm{D}, \mathrm{DK}$, and $\mathrm{PB}$ contributed to the design and implementation of the research; $\mathrm{ZV}, \mathrm{ZV}, \mathrm{EF}, \mathrm{VP}$ and $\mathrm{ZR}$ participated in data acquisition and analysis; AC performed the image analysis, $\mathrm{BD}$ performed the statistical analysis; JO helped in data evaluation, $\mathrm{KH}$, $\mathrm{BD}$ and $\mathrm{ZV}$ wrote the article. All Authors were subsequently involved in data interpretation. All Authors revised and approved the article.

\section{Acknowledgements}

This project was supported by Analytic Healthcare Quality User Information Program of the National Research, Development and Innovation Fund, Hungarian Government, Grant VKSZ 12-1-20130012 and by the Szeged Foundation for Cancer Research.

\section{References}

1 Thibouw D, Truc G, Bertaut A, Chevalier C, Aubignac L and Mirjolet C: Clinical and dosimetric study of radiotherapy for glioblastoma: three-dimensional conformal radiotherapy versus intensity-modulated radiotherapy. J Neurooncol 137(2): 429-438, 2018. PMID: 29374810. DOI: 10.1007/s11060-017-2735-y

2 Briere TM, McAleer MF, Levy LB and Yang JN: Sparing of normal tissues with volumetric arc radiation therapy for glioblastoma: Single-institution clinical experience. Radiat Oncol 12(1): 79, 2017. PMID: 28464840. DOI: 10.1186/s13014017-0810-3

3 Exeli AK, Kellner D, Exeli L, Steininger P, Wolf F, Seldmayer $\mathrm{F}$ and Deutschmann H: Cerebral cortex dose sparing for glioblastoma patients: IMRT versus robust treatment planning. Radiat Oncol 13(1): 20, 2018. PMID: 29409516. DOI: 10.1186/s13014-018-0953-x

4 Petr J, Platzek I, Hofheinz F, Mutsaerts HJMM, Asllani I, van Osch MJP, Seidlitz A, Krukowski P, Gommlich A, BeuthienBaumann B, Jentsch C, Maus J, Troost EGC, Baumann M, Krause $\mathrm{M}$ and van den Hoff J: Photon vs. proton radiochemotherapy: Effects on brain tissue volume and perfusion. Radiother Oncol 128(1): 121-127, 2018. PMID: 29370984. DOI: 10.1016/j.radonc.2017.11.033

5 Mehta S, Gajjar SR, Padgett KR, Asher D, Stoyanova R, Ford JC and Mellon EA: Daily tracking of glioblastoma resection cavity, cerebral edema, and tumor volume with MRI-guided radiation therapy. Cureus 10(3): e2346, 2018. PMID: 29796358. DOI: $10.7759 /$ cureus.2346
6 Zhang L, Wang Z, Shi C, Long T and Xu XG: The impact of robustness of deformable image registration on contour propagation and dose accumulation for head and neck adaptive radiotherapy. J Appl Clin Med Phys 19(4): 185-194, 2018. PMID: 29851267. DOI: 10.1002/acm2.12361

7 Saito N, Schmitt D and Bangert M: Correlation between intrafractional motion and dosimetric changes for prostate IMRT: Comparison of different adaptive strategies. J Appl Clin Med Phys 19(4): 87-97, 2018. PMID: 29862644. DOI: 10.1002/acm2.12359

8 Matsuo Y: A promising result of locoregional tumor control with biologically adaptive RT in patients with locally advanced nonsmall cell lung cancer. Transl Lung Cancer Res 7(2): 111-113, 2018. PMID: 29780704. DOI: 10.21037/tlcr.2018.03.01

9 Tsien C, Gomez-Hassan D, Ten Haken RK, Tatro D, Junck L, Chenevert TL and Lawrence T: Evaluating changes in tumor volume using magnetic resonance imaging during the course of radiotherapy treatment of high-grade gliomas: implications for conformal doseescalation studies. Int J Radiat Oncol Biol Phys 62(2): 328-332, 2005. PMID: 15890571. DOI: 10.1016/j.ijrobp.2004.10.010

10 Kim TG and Lim DH: Interfractional variation of radiation target and adaptive radiotherapy for totally resected glioblastoma. J Korean Med Sci 28(8): 1233-1237, 2013. PMID: 23960453. DOI: $10.3346 / \mathrm{jkms} .2013 .28 .8 .1233$

11 Grossman R, Shimony N, Shir D, Gonen T, Sitt R, Kimchi TJ, Harosh CB and Ram Z: Dynamics of FLAIR volume changes in glioblastoma and prediction of survival. Ann Surg Oncol 24(3): 794-800, 2017. PMID: 27766560. DOI: 10.1245/s10434-016$5635-\mathrm{z}$

12 Champ CE, Siglin J, Mishra MV, Shen X, Werner-Wasik M, Andrews DW, Mayekar SU, Liu H and Shi W: Evaluating changes in radiation treatment volumes from post-operative to same-day planning MRI in high-grade gliomas. Radiat Oncol 7: 220, 2012. PMID: 23259933. DOI: 10.1186/1748-717X-7-220

13 Liang HT, Chen WY, Lai SF, Su MY, You SL, Chen LH, Tseng HM, Chen CM, Kuo SH and Tseng WI: The extent of edema and tumor synchronous invasion into the subventricular zone and corpus callosum classify outcomes and radiotherapy strategies of glioblastomas. Radiother Oncol 125(2): 248-257, 2017. PMID: 29056290. DOI: 10.1016/j.radonc.2017.09.024

14 Chatterjee A, Serban M, Abdulkarim B, Panet-Raymond V, Souhami L, Shenouda G, Sabri S, Jean-Claude B and Seuntjens J: Performance of knowledge-based radiation therapy planning for the glioblastoma disease site. Int J Radiat Oncol Biol Phys 99(4): 10211028, 2017. PMID: 28870791. DOI: 10.1016/j.jjrobp.2017.07.012

15 Hofmaier J, Kantz S, Söhn M, Dohm OS, Bächle S, Alber M, Parodi M, Belka C and Niyazi M: Hippocampal sparing radiotherapy for glioblastoma patients: A planning study using volumetric modulated arc therapy. Radiat Oncol 11(1): 118, 2016. PMID: 27609371. DOI: 10.1186/s13014-016-0695-6

16 Mann J, Ramakrishna R, Magge R and Wernicke AG: Advances in Radiotherapy for Glioblastoma. Front Neurol 8: 748, 2018. PMID: 29379468. DOI: 10.3389/fneur.2017.00748

17 Niyazi M, Geisler J, Siefert A, Schwarz SB, Ganswindt U, Garny S, Schnell O, Suchorska B, Kreth FW, Tonn JC, Bartenstein P, la Fougère $\mathrm{C}$ and Belka $\mathrm{C}$ : FET-PET for malignant glioma treatment planning. Radiother Oncol 99(1): 44-8, 2011. PMID: 21458093. DOI: $10.1016 /$ j.radonc.2011.03.001

18 Ghose A, Lim G and Husain S: Treatment for GBM: Current guidelines and Canadian practice. Curr Oncol 17(6): 52-58, 2010. PMID: 21151410 . DOI: $10.3747 /$ co.v17i6.574 
19 Tsien C, Moughan J, Michalski JM, Gilbert MR, Purdy J, Simpson J, Kresel JJ, Curran WJ, Diaz A and Mehta MP: Phase I three-dimensional conformal radiation dose escalation study in newly diagnosed glioblastoma: Radiation Therapy Oncology Group Trial 98-03. Int J Radiat Oncol Biol Phys 73(3): 699-708, 2009. PMID: 18723297. DOI: 10.1016/j.ijrobp.2008.05.034

20 Cabrera AR, Kirkpatrick JP, Fiveash JB, Shih HA, Koay EJ, Lutz S, Petit J, Chao ST, Brown PD, Vogelbaum M, Reardon DA, Chakravarti A, Wen PY and Chang E: Radiation therapy for glioblastoma: executive summary of an American Society for Radiation Oncology evidence-based clinical practice guideline. Pract Radiat Oncol 6(4): 217-225, 2016. PMID: 27211230. DOI: 10.1016/j.prro.2016.03.007

21 Niyazi M, Brada M, Chalmers AJ, Combs SE, Erridge SC, Fiorentino A, Grosu AL, Lagerwaard FJ, Minniti G, Mirimanoff RO, Ricardi U, Short SC, Weber DC and Belka C: ESTROACROP guideline "target delineation of glioblastomas". Radiother Oncol 118(1): 35-42, 2016. PMID: 26777122. DOI: 10.1016/j.radonc.2015.12.003

22 Paulsson AK, McMullen KP, Peiffer AM, Hinson WH, Kearns WT, Johnson AJ, Lesser GJ, Ellis TL, Tatter SB, Debinski W, Shaw EG and Chan MD: Limited margins using modern radiotherapy techniques does not increase marginal failure rate of glioblastoma. Am J Clin Oncol 37(2): 177-181, 2014. PMID: 23211224. DOI: 10.1097/COC.0b013e318271ae03

23 Gupta T, Nair V, Paul SN, Kannan S, Moiyadi A, Epari S and Jalali R: Can irradiation of potential cancer stem-cell niche in the subventricular zone influence survival in patients with newly diagnosed glioblastoma? J Neurooncol 109(1): 195-203, 2012. PMID: 22555992. DOI: 10.1007/s11060-012-0887-3

24 Lee P, Eppinga W, Lagerwaard F, Cloughesy T, Slotman B, Nghiemphu PL, Wang PC, Kupelian P, Agazaryan N, Demarco J, Selch MT, Steinberg M and Kang JJ: Evaluation of high ipsilateral subventricular zone radiation therapy dose in glioblastoma: A pooled analysis. Int J Radiat Oncol Biol Phys 86(4): 609-615, 2013. PMID: 23462418. DOI: 10.1016/j.ijrobp.2013.01.009

25 Chen L, Guerrero-Cazares H, Ye X, Ford E, McNutt T, Kleinberg L, Lim M, Chaichana K, Quinones-Hinojosa A and Redmond K: Increased subventricular zone radiation dose correlates with survival in glioblastoma patients after gross total resection. Int J Radiat Oncol Biol Phys 86(4): 616-622, 2013. PMID: 23540348. DOI: 10.1016/j.ijrobp.2013.02.014

26 Elicin O, Inac E, Uzel EK, Karacam S and Uzel OE: Relationship between survival and increased radiation dose to subventricular zone in glioblastoma is controversial. J Neurooncol 118(2): 413-419, 2014. PMID: 24668610. DOI: $10.1007 / \mathrm{s} 11060-014-1424-3$

27 Anker CJ, Bagshaw HP, Sarkar V, Dritto M, Boucher K, Jensen RL and Shrieve DC: Impact of subventricular zone dose and relationship to glioblastoma tumor location on outcomes. Int J Radiat Oncol Biol Phys 93(3): 110-111, 2015. DOI: 10.1016/j.ijrobp.2015.07.829

28 Khalifa J, Tensaouti F, Lusque A, Plas B, Lotterie JA, Benouaich-Amiel A, Uro-Coste E, Lubrano V and CohenJonathan Moyal E: Subventricular zones: New key targets for glioblastoma treatment. Radiat Oncol 12(1): 67, 2017. PMID: 28424082. DOI: 10.1186/s13014-017-0791-2

29 Darázs B, Ruskó L, Végváry Z, Ferenczi L, Dobi Á, Paczona V, Varga Z, Fodor E and Hideghéty K: Subventricular zone volumetric and dosimetric changes during postoperative brain tumor irradiation and its impact on overall survival. Phys Med 68: 35-40. PMID: 31733404. DOI: 10.1016/j.ejmp.2019.10.039

30 Kruser TJ, Bosch WR, Badiyan SN, Bovi JA, Ghia AJ, Kim MM, Solanki AA, Sachdev S, Tsien C, Wang TJC, Mehta MP and McMullen KP: NRG Brain Tumor Specialists Consensus Guidelines for Glioblastoma Contouring. J Neurooncol 143(1): 157166, 2019. PMID: 30888558. DOI: 10.1007/s11060-019-03152-9.

31 Ho KF, Marchant T, Moore C, Webster G, Rowbottom C, Penington H, Lee L, Yap B, Sykes A and Slevin N: Monitoring dosimetric impact of weight loss with kilovoltage $(\mathrm{kV})$ cone beam CT (CBCT) during parotid-sparing IMRT and concurrent chemotherapy. Int J Radiat Oncol Biol Phys 82(3): 375-382, 2012. PMID: 22197229. DOI: 10.1016/j.ijrobp.2011.07.004

32 Brouwer C, Steenbakkers R, Langendijk J and Sijtsema N: Idenitfying patients who may benefit from adaptive radiotherapy: Does the literature on anatomic and dosimetric changes in head and neck organs at risk during radiotherapy provide information to help? Radiother Oncol 115(3): 285-294, 2015. PMID: 26094076. DOI: 10.1016/j.radonc.2015.05.018.

33 Lim-Reinders S, Keller BM, Al-Ward S, Sahgal A and Kim A: Online adaptive radiation therapy. Int J Radiat Oncol Biol Phys 99(4): 994-1003, 2017. PMID: 28916139. DOI: 10.1016/j.ijrobp. 2017.04.023.

34 Robar JL, Day A, Clancey J, Kelly R, Yewondwossen M, Hollenhorst H, Rajaraman M and Wilke D: Spatial and dosimetric variability of organs at risk in head-and-neck intensity-modulated radiotherapy. Int J Radiat Oncol Biol Phys 68(4): 1121-1130, 2007. PMID: 17398025. DOI: 10.1016/j.jijrobp.2007.01.030.

35 Marzi S, Pinnaro P, D’Alessio D, Strigari L, Bruzzaniti V, Giordano C, Giovinazzo G and Marucci L: Anatomical and dose changes of gross tumour volume and parotid glands for head and neck cancer patients during Intenisty-modulated radiotherapy: effect on the probability of xerostomia incidence. Clin Oncol 24(3): e54-62, 2012. PMID: 22138192. DOI: 10.1016/j.clon.2011.11.006.

36 Surucu M, Shah KK, Roeske JC, Choi M, Small Jr W and Emami B: Adaptive radiotherapy for head and neck cancer: Implications for clinical and dosimetry outcomes. Technol Cancer Res Treat 16(2): 218-223, 2017. PMID: 27502958. DOI: $10.1177 / 1533034616662165$

37 Ruschin ME, Sahgal A, Chugh B, Lau A, Tseng CL, Myrehaug $\mathrm{S}$ and Lee Y: Preliminary investigation of adaptive glioblastoma radiation therapy using the integrated high-field MRI-LINAC. Int J Radiat Oncol Biol Phys 99(2): 717, 2017. DOI: 10.1016/j.ijrobp.2017.06.2328

38 Muruganandham M, Clerkin PP, Smith BJ, Anderson CM, Morris A, Capizzano AA, Magnotta V, McGuire SM, Smith MC, Bayouth JE and Buatti JM: 3-dimensional magnetic resonance spectroscopic imaging at 3-Tesla for early response assessment of glioblastoma patients during external beam radiation therapy. Int J Radiat Oncol Biol Phys 90(1): 181-189, 2014. PMID: 24986746. DOI: $10.1016 /$ j.ijrobp.2014.05.014

Received June 2, 2020

Revised June 24, 2020

Accepted June 27, 2020 